\title{
Investigating media that support red wolf (Canis rufus) sperm viability and capacitation in vitro
}

\author{
Jennifer B Nagashima1,*, Marcia de Almeida Monteiro Melo Ferraz ${ }^{1,2, *}$, Sarah H Kamen ${ }^{1,3}$ and Nucharin Songsasen ${ }^{1}$ \\ ${ }^{1}$ Smithsonian Conservation Biology Institute, National Zoological Park, Front Royal, Virginia, USA \\ 2Gene Center, Ludwig-Maximilians University, Munich, Bayern, Germany \\ 3Department of Biological and Environmental Sciences, Longwood University, Farmville, Virginia, USA
}

Correspondence should be addressed to J B Nagashima: nagashimaj@si.edu

*(J B Nagashima and M d A M M Ferraz contributed equally to this work)

\begin{abstract}
The red wolf is a critically endangered canid, with $\sim 250$ and $~ 20$ individuals in the ex situ and reintroduced wild populations, respectively. Assisted reproductive technologies such as sperm cryopreservation and in vitro fertilization therefore represent critically-needed tools to manage these populations. However, the motility of post-thaw red wolf sperm rapidly declines during in vitro incubation, hindering the ability to develop these technologies. In this study, we evaluated the influence of several culture media (a modified canine capacitation medium (mCCM), a modified North Carolina State University-23 medium (mNCSU-23), a synthetic oviductal fluid (SOF), a fertilization Tyrode's medium base or Fert-TALP (FERT), and a TRISbased buffer (TRIS)) on the survival and capacitation of red wolf sperm during extended ( $18 \mathrm{~h}$ ) incubation at $38.5^{\circ} \mathrm{C}$ and $5 \%$ $\mathrm{CO}_{2}$. Red wolf sperm motility averaged ( \pm S.E.M.) $73.8 \pm 7.1 \%$ at the time of collection, and was better maintained over $4 \mathrm{~h}$ incubation in mCCM $(55.0 \pm 9.8 \%)$ and mNCSU-23 (54.7 \pm 10.4$)$, compared to mSOF (43.8 $\pm 8.3 \%)$, FERT (30 \pm 10.5$)$, and TRIS (16.4 $\pm 4.1 \%)$ solutions. Patterns of tyrosine phosphorylation signal, as assessed via immunocytochemistry, indicated induction of capacitation between 2 and $4 \mathrm{~h}$ in vitro culture. Tyrosine phosphorylation signal was particularly robust in mCCM and mNCSU-23 incubated sperm, although significant acrosome exocytosis was not observed in response to progesterone supplementation after $3 \mathrm{~h}$ incubation in any of the media. In sum, results indicate mCCM and mNCSU-23 are promising base media for the in vitro incubation and capacitation of red wolf sperm, for assisted reproduction applications.
\end{abstract}

\section{Lay summary}

Development of assisted reproductive technologies such as in vitro fertilization and artificial insemination is of high importance to the genetic management of critically endangered species such as the red wolf (Canis rufus). However, these technologies require the ability to maintain sperm viability and function during extended incubation, which has not been successful for the red wolf thus far. In this study, various culture media developed for sperm/egg/embryo culture in large mammalian species were evaluated for their ability to maintain red wolf sperm motility under physiological incubation conditions. Media and conditions previously utilized for domestic dog sperm were found to best support sperm incubation and capacitation (process of becoming competent to fertilize an egg) in the red wolf, representing a key step for future development of assisted reproductive technologies for the species.

Key Words: - sperm $\quad$ capacitation $\quad$ red wolf $\quad$ assisted reproduction

(C) 2020 The authors https://doi.org/10.1530/RAF-20-0042
Published by Bioscientifica Ltd

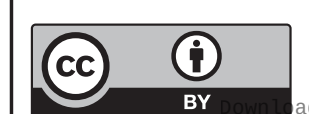




\section{Introduction}

The red wolf is a critically endangered American canid, with fewer than 20 individuals in the reintroduced population and approximately 250 managed individuals in the Association of Zoos and Aquariums (AZA) Species Survival Plan population (Lasher 2020). Specifically, the most recent red wolf Population Viability Analysis determined that, in the absence of ongoing genetic management or population expansion, the AZA SSP population will retain only $\sim 75 \%$ gene diversity in the next 125 years, and the reintroduced population is projected to go extinct over the next 8-37 years (Faust et al. 2016). As result, it is vital to the long-term survival of the species that every valuable animal is represented in the next generation, to maintain genetic variability. While ideally this could be accomplished via natural breeding, this comes with the risk of transporting individuals to different locations for breeding and failed reproduction due to sexual incompatibility. In recent decades, assisted reproductive technologies (ART), especially artificial insemination (AI) have played significant roles in the management of many endangered species, including the black-footed ferret (Howard et al. 2016), the giant panda (Huang et al. 2012) and whooping crane (Blanco et al. 2009). Though not yet regularly implemented in canid breeding management programs, there have been successful reports of artificial insemination in gray wolves (Asa et al. 2006), blue and red foxes (Nyberg 1980, Farstad et al. 1992), and critically endangered Mexican gray wolves (Mossotti 2017). As such, there is significant interest and potential for AI and other ART to support endangered canid conservation efforts (Comizzoli et al. 2009, Amstislavsky et al. 2012).

Previous assisted reproduction research in red wolves has characterized semen and optimized sperm cryopreservation and thawing protocols (Goodrowe et al. 1998, Koehler et al. 1998, Franklin et al. 2018, Ferraz et al. 2020). For example, though the acrosomal membranes of red wolf sperm are highly sensitive to cryodamage (Goodrowe et al. 1998), investigations on cooling strategies (Goodrowe et al. 2001), extender osmolarity and egg yolk concentration (Lockyear et al. 2009), and cryoprotectant and post-thaw culture temperatures (Franklin et al. 2018), have improved survival and motility post-thaw. However, sperm motility/viability has been shown to drop precipitously during post-thaw incubation in the TRIS-based extender commonly used in cryopreservation (Lockyear et al. 2009, Franklin et al. 2018). As such, improvements in sperm incubation conditions and/or cryopreservation methods are necessary for future use of banked semen. Specifically, our ability to fully evaluate the quality and longevity of post-thaw semen as well as to develop in vitro fertilization technologies for this species requires long-term sperm incubation capacity.

Similarly, additional studies into the fertilizing capacity of red wolf sperm, in general, are necessary. Thus far, only one study has described the binding and penetration of fresh and frozen/thawed red wolf sperm to domestic dog oocytes, as an assay of the fertilizing potential of red wolf sperm (Goodrowe et al. 2001). In vivo, ejaculated sperm are not immediately capable of fertilizing an oocyte, and first must undergo capacitation. Capacitation is the process by which a sperm becomes developmentally competent to fertilize (Chang 1951, Austin 1952) and is characterized by phosphorylation of tyrosine residues in sperm proteins, the development of hyperactive motility, and the ability of the sperm to undergo acrosomal exocytosis (Visconti et al. 1995). Once in the female reproductive tract, sperm are exposed to stimuli that support efflux of sterols from the plasma membrane, removal of decapacitating seminal plasma proteins, and changes in intracellular $\mathrm{pH}$ and calcium, which cumulatively promote capacitation (Visconti et al. 1995). In vitro capacitation can be stimulated by compounds such as BSA, which results in cholesterol efflux from the sperm membrane and increased membrane fluidity (Cross 1998). Sodium bicarbonate, which facilitates the rise of sperm cyclic adenosine 3',5'-monophosphate (cAMP) and intracellular pH (Visconti et al. 1999, Demarco et al. 2003), supports protein tyrosine phosphorylation, lipid membrane remodeling, and acrosome exocytosis (Buffone et al. 2014). Sperm subsequently exposed to factors present in the oviductal environment, including progesterone or zona pellucida proteins, undergo acrosomal exocytosis in vitro if capacitation has been achieved (Roldan et al. 1994, Brewis et al. 2001). This acrosome reaction, in turn, is necessary for sperm penetration and fusion with the oocyte and fertilization (Wassarman 1999). Thus, in vitro sperm incubation and capacitation methods are key to evaluate the fertilizing capacity of red wolf semen, and for the future development of IVF in the species.

Toward these goals, a variety of 'capacitating' and gamete/embryo holding media have been developed for other species which can be evaluated for utility in supporting red wolf sperm in vitro. Domestic dog IVF utilized a modified canine capacitation medium (mCCM) (Nagashima et al. 2015), which had been developed specifically to promote sperm capacitation (Mahi \& Yanagimachi 1978). Fert-TALP (FERT), a modified Tyrode's balanced salt solution, is traditionally used in bovine IVF 
(Parrish 2014). A medium developed for porcine embryo culture, named North Carolina State University-23 (NCSU-23 (Petters \& Wells 1993)), has also been utilized for IVF in the domestic dog (Nagashima et al. 2015). In the latter study, a modified NCSU-23 containing magnesium chloride and sodium pyruvate maintained dog sperm viability in vitro for over $24 \mathrm{~h}$ (Nagashima, personal observation). Synthetic oviductal fluid (SOF), was originally developed for ovine embryo work, but has also been utilized in domestic dog oocyte culture (Hewitt \& England 1999). Finally, TRIS extender, the medium used in red wolf sperm cryopreservation, has also been utilized in the post-thaw incubation of red wolf semen (Lockyear et al. 2009, Franklin et al. 2018). Importantly, incubation of both fresh (Goodrowe et al. 1998) and frozen-thawed (Franklin et al. 2018) red wolf sperm in TALP- and TRIS-based media, respectively, at $37^{\circ} \mathrm{C}$ resulted in a more rapid decline of sperm motility/viability, compared with ambient temperatures. Therefore, the objective of this study is to identify a base media (mCCM, FERT, mNCSU-23, mSOF, or TRIS) supportive of red wolf sperm incubation at physiological temperatures $\left(38.5^{\circ} \mathrm{C}\right)$, toward the goal of developing a capacitation protocol for red wolf sperm.

\section{Materials and methods}

\section{Animals}

Adult (ages 2-9 years old) male red wolves housed at AZA Species Survival Plan facilities in the Eastern United States were selected for collection based on health status, mean kinship value, and geographic location. Animals were exposed to natural photoperiod, fed diets consisting of commercially available dry dog food and/or whole carcass, with water provided ad libitum. All procedures were done in accordance with the AZA SSP and USFWS guidelines and Smithsonian Institution International Animal Care and Use Committee approval (IACUC \#18-05).

\section{Sperm collection and processing}

A single collection attempt was made per animal in the 2018 and 2019 breeding seasons (January-March), for a total of eight ejaculates collected from six individuals (aged 3-9 years, with two wolves collected in both 2018 and 2019 seasons). Wolves were fasted $24 \mathrm{~h}$ prior to semen collection. On the day of semen collection, animals were captured, transferred to a crate and transported a short distance from their outdoor enclosure to an indoor treatment room, wherein wolves were anesthetized by veterinary staff. Typically, red wolves were anesthetized using butorphanol $(0.4 \mathrm{mg} / \mathrm{kg}$ body weight $\mathrm{IM})$ and medetomidine $(0.4 \mathrm{mg} / \mathrm{kg} \mathrm{IM})$. Prior to the start of electroejaculation, the rectum was evacuated using a well-lubricated gloved hand and the penis carefully exteriorized, washed thoroughly with sterile saline and wiped dry.

Red wolf semen was collected via electroejaculation as previously described (Franklin et al. 2018). Briefly, to avoid urine contamination in semen samples, the urinary bladder was emptied by inserting a $5 \mathrm{Fr}, 55.8 \mathrm{~cm}$ long polypropylene catheter (Covidien/Kendall) into the urethra, the urine aspirated and the bladder flushed with warmed sterile saline. Thereafter, a lubricated rectal probe $(1.9-2.1 \mathrm{~cm})$ was inserted and 60-90 electrical stimulations (2-6 V) were applied over a period of $20 \mathrm{~min}$. The intensity of the stimulation was adjusted as appropriate based on the individual animal's physical response. Ejaculates were collected into sterile polypropylene specimen cups, pooled, and a small volume was assessed for sperm concentration (via hemocytometer), motility, and $\mathrm{pH}$. In cases where semen volume or concentration was low, the second round of stimulations was attempted. Animals were allowed to recover from anesthesia under the care of veterinary staff.

Semen fractions with no evidence of urine contamination (based on color and $\mathrm{pH}$ 6-8) and presence of sperm from all stimulation rounds were pooled per individual, then centrifuged at $500 \boldsymbol{g}$ for $3 \mathrm{~min}$ to pellet the sample/remove seminal plasma. A portion of the sperm was then resuspended/washed in $1 \mathrm{~mL}$ warmed sterile PBS (Sigma-Aldrich 59331C) prior to in vitro incubation and capacitation evaluations.

\section{Sperm incubation}

Following collection and washing, sperm were incubated in each of five different base media at a final concentration of $5 \times 10^{6} \mathrm{sperm} / \mathrm{mL}$ at $38.5^{\circ} \mathrm{C}$ and $5 \% \mathrm{CO}_{2}$. The five media included: mCCM (Nagashima et al. 2015), FertTALP (Ferraz et al. 2018), mSOF (Hewitt \& England 1999), modified NCSU-23 (mNCSU-23 (Petters 1992)), and Tris buffer (Franklin et al. 2018) without egg yolk (TRIS) (Supplementary Table 1, see section on supplementary materials given at the end of this article). All media were adjusted to $\sim 300$ mOsm (range 295-311 mOsm) and a $\mathrm{pH}$ of $7.75-7.8$. At $3 \mathrm{~h}$ incubation, $6 \mu \mathrm{g} / \mathrm{mL}$ progesterone (or $19 \mu \mathrm{M}$, in DMSO, Sigma-Aldrich P8783) was added

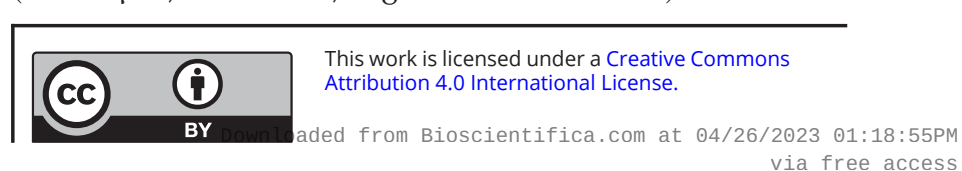


as a physiological stimulant of acrosome exocytosis (Brewis et al. 2001). Incubations were performed in fourwell dishes (NUNC, Fisher Scientific). Motility evaluations (\% of sperm with a flagellar beating from at least five areas, as previously described (Ferraz et al. 2020) were performed on washed sperm at $0,1,2,4$, and $18 \mathrm{~h}$. Dishes were gently swirled to mix and detach sperm from the dish, then $\sim 5 \mu \mathrm{L}$ of the sample was pipetted onto glass slides with a coverslip and evaluated via Brightfield Microscopy (Olympus BX41). The appearance of sperm with hyperactive motility patterns (asymmetrical, rapid flagellar beating (Yanagimachi 1970)) was noted in the treatment groups/time points observed, but could not be accurately quantified due to adherence of sperm heads to the glass slides. At the $0,1,2,4$ and $18 \mathrm{~h}$ time points, an additional $5 \mu \mathrm{L}$ of the sample were removed and fixed with $4 \%$ paraformaldehyde, and stored at $4^{\circ} \mathrm{C}$ until evaluation via fluorescence microscopy for acrosome and tyrosine phosphorylation statuses.

In a subset of the animals $(n=5)$, sperm were additionally incubated in either mNCSU-23 or in mNCSU-23 containing only $1 \mathrm{mg} / \mathrm{mL}$ BSA without sodium bicarbonate, with osmolarity returned to 300 mOsm with $\mathrm{NaCl}$, to reduce capacitation-stimulation (named non-capacitating mNCSU-23, or NC-mNCSU-23). After $3 \mathrm{~h}$ incubation, sperm in each medium were either exposed to $6 \mu \mathrm{g} / \mathrm{mL}$ progesterone (P4) or DMSO vehicle control. Sperm motility, acrosome status, and tyrosine phosphorylation patterns were evaluated as described earlier at hours $1,2,4$, and 18 of incubation at $38.5^{\circ} \mathrm{C}$ in $5 \% \mathrm{CO}_{2}$.

\section{Fluorescence microscopy}

Sperm samples were stained for acrosome (FITC-PNA, ThermoFisher L21409), tyrosine phosphorylation (antiphosphotyrosine, Millipore Sigma 05-321), and nuclear (Hoechst 33342, Invitrogen H3570) evaluations. Briefly, fixed sperm samples were smeared on glass slides and dried at room temperature. Following three PBS washes, slides were permeabilized with $0.1 \%$ Triton-X (SigmaAldrich X100) in PBS then blocked in 1\% BSA in 0.1\% Triton-X PBS for $1 \mathrm{~h}$. Slides were incubated with $2 \mu \mathrm{g} / \mathrm{mL}$ anti-phosphotyrosine antibody in a humidified chamber, either overnight at $4^{\circ} \mathrm{C}$ or for $4 \mathrm{~h}$ at room temperature. Samples were incubated with 1:200 dilution of Alexa rabbit anti-mouse 568, $10 \mu \mathrm{g} / \mathrm{mL}$ FITC-PNA and $5 \mu \mathrm{g} / \mathrm{mL}$ Hoechst 33342 at room temperature for $1 \mathrm{~h}$. After three PBS washes, glass coverslips were mounted with Prolong Anti-fade mountant (ThermoFisher P36980) and slides were observed at $40 \times$ magnification with an EVOS Fl Auto 2 (ThermoFisher).

At least 90 sperm were counted for each time point and treatment, per animal. Acrosome status was recorded as intact, partially-intact, or reacted. Localization of phosphorylated tyrosine signal was recorded as in the head, midpiece, tail, tail and midpiece, all, or none of the sperm (Supplementary Fig. 1).

\section{Statistical analyses}

All data were analyzed in R Studio (v. 1.3.1056), with significance set at $P<0.05$. Motility data (\% motile sperm) were evaluated with a nonparametric Wilcoxon test. Tyrosine phosphorylation pattern data were subjected to linear transformation then evaluated via generalized linear mixed effect model with gamma distribution (link log) in package 'Ime4' (ver. 1.1-23) (Bates et al. 2014). Percent reacted acrosome data were logit transformed to achieve normality, then evaluated via linear mixed effect model with r package 'nlme' (ver. 3.1-141) (Pinheiro et al. 2012). Factors included incubation time, treatment group, the interaction between the treatment group and incubation time, and individual wolf (random factor). For analyses of non-capacitating and capacitating mNCSU-23 data in the presence/absence of $\mathrm{P} 4$ and the interaction between treatment group and P4 were also included as fixed effects. Post hoc evaluations were done with a nonparametric Wilcoxon test.

\section{Results}

Semen collected via electroejaculation from red wolves ranged from 1.1 to $10.3 \mathrm{~mL}$ with a concentration of 18 to $198 \times 10^{6} \mathrm{sperm} / \mathrm{mL}$ and $30-90 \%$ total motile (Table 1). Over the incubation period, sperm motility was better maintained in mCCM and mNCSU-23 compared with FERT and TRIS (Fig. 1A). For example, after $4 \mathrm{~h}$ incubation, percent motility (mean \pm s.e.) averaged $55.0 \pm 9.8$ in mCCM and $54.7 \pm 10.4$ mNCSU-23, respectively, compared with $30 \pm 10.5$ in FERT and $16.4 \pm 4.1 \%$ in TRIS. While sperm attachment to glass slides prevented quantification of sperm hyperactive motility, characteristic star-spin movement and lateral head displacement with rapid flagellar beating were observed at the $4 \mathrm{~h}$ time point only for mCCM, mNCSU-23 (Supplementary Video 1), and mSOF-treated sperm. By $18 \mathrm{~h}$ incubation, mNCSU23 incubated sperm displayed higher motility than those incubated in TRIS $(P<0.05)$, but was not significantly

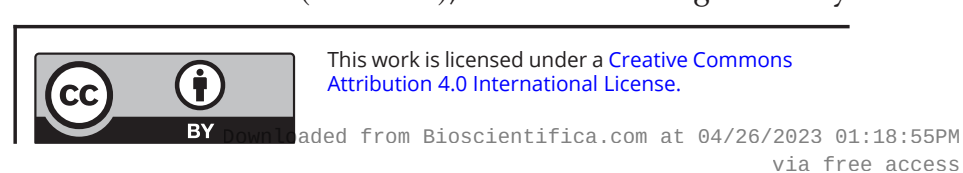


Table 1 Summary of semen characteristics following electroejaculation in adult male red wolves.

\begin{tabular}{|c|c|c|c|}
\hline Collection year & Wolf ID & Age & Volume $(\mu \mathrm{L})$ \\
\hline 2018 & 2119 & 3 & 1056 \\
\hline 2018 & 2118 & 3 & 2000 \\
\hline 2018 & 2075 & 4 & 2800 \\
\hline 2019 & $2208 *$ & 2 & 2000 \\
\hline 2019 & $2206 *$ & 2 & 4310 \\
\hline 2019 & $2119 *$ & 4 & 2500 \\
\hline 2019 & $2118 *$ & 4 & 10,300 \\
\hline 2019 & $1790 *$ & 9 & 5000 \\
\hline Mean & & & 3745.75 \\
\hline S.E.M. & & & 1040.48 \\
\hline
\end{tabular}

\begin{tabular}{c}
\hline $\mathbf{p H}$ \\
\hline 6.8 \\
$6.8-7.9$ \\
$6.8-7.9$ \\
7.5 \\
7 \\
$\mathrm{NA}$ \\
$7-7.5$ \\
$6-6.5$ \\
7.04 \\
0.15 \\
\hline
\end{tabular}

\begin{tabular}{c}
\hline Concentration $($ sperm/mL) \\
\hline $18 \times 10^{6}$ \\
$28 \times 10^{6}$ \\
$198 \times 10^{6}$ \\
$25 \times 10^{6}$ \\
$25 \times 10^{6}$ \\
$34 \times 10^{6}$ \\
$59 \times 10^{6}$ \\
$190.5 \times 10^{6}$ \\
$72.2 \times 10^{6}$ \\
$27.0 \times 10^{6}$ \\
\hline
\end{tabular}

$\begin{array}{r}\hline \text { Total sperm } \\ \hline 19 \times 10^{6} \\ 56 \times 10^{6} \\ 554 \times 10^{6} \\ 50 \times 10^{6} \\ 108 \times 10^{6} \\ 85 \times 10^{6} \\ 608 \times 10^{6} \\ 952 \times 10^{6} \\ 304 \times 10^{6} \\ 125 \times 10^{6} \\ \hline\end{array}$

\begin{tabular}{c}
\hline \% motility \\
\hline 75 \\
70 \\
80 \\
30 \\
65 \\
90 \\
90 \\
90 \\
73.75 \\
7.12
\end{tabular}

*Subset of samples which were also assessed under non-capacitating conditions.
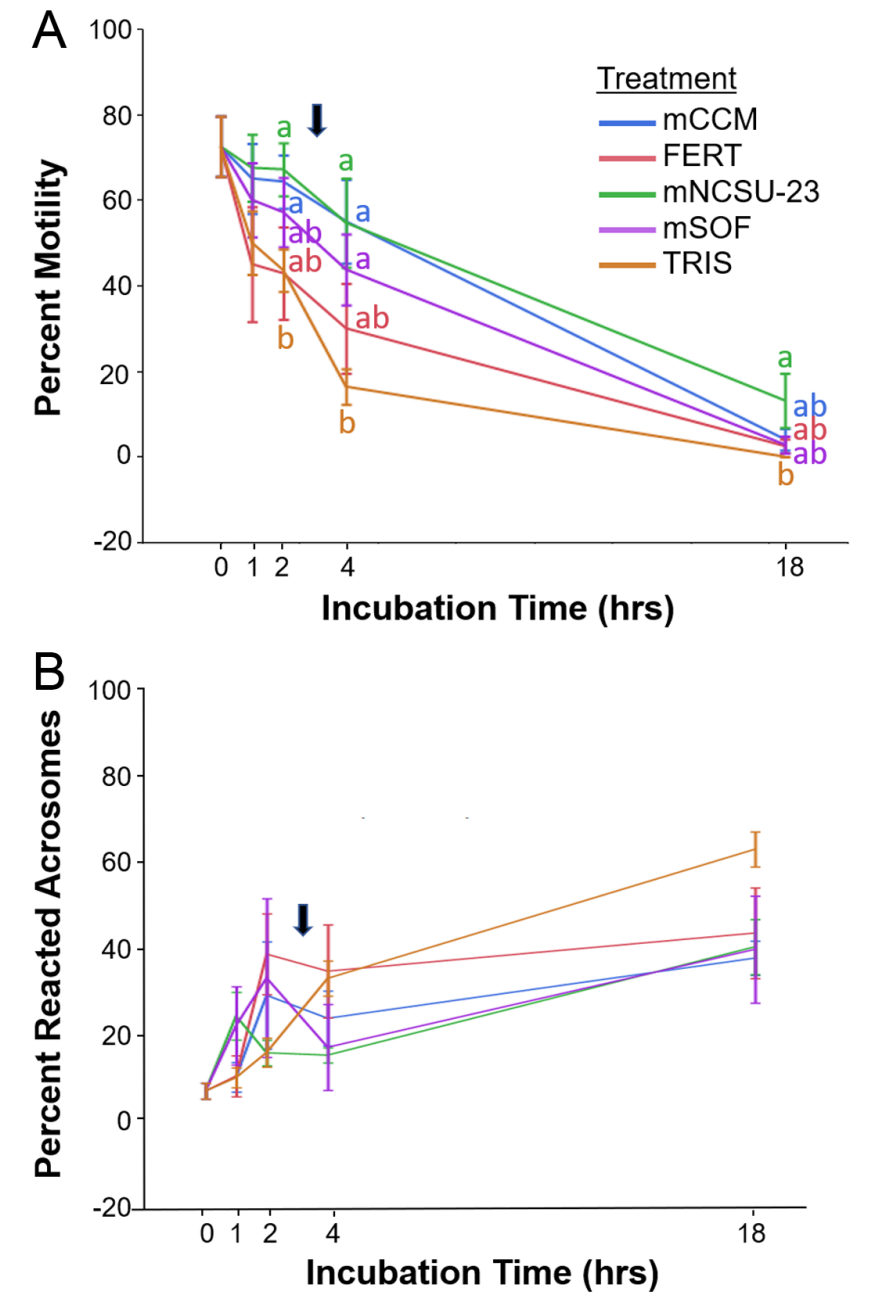

Figure 1 Motility and acrosome status (mean \pm S.E.M.) of red wolf sperm in mCCM, FERT, mNCSU-23, mSOF, and TRIS after 1, 2, 4, and $18 \mathrm{~h}$ incubation, with (A) percent motile sperm, and (B) percentage of sperm with reacted acrosomal membranes. Letters indicate significant differences between treatments for each time point $(P<0.05)$ and black arrow denotes time of progesterone treatment. different from mCCM, mSOF, or FERT incubated sample $(P>0.05)$.

Red wolf sperm acrosomes were evaluated at times $0,1,2,4$, and 18 for each wolf and treatment group. Only incubation time $(P<0.01)$, not treatment group, was significantly based on the model. Overall, the proportion of sperm with variable patterns of tyrosine phosphorylation (PY) immunofluorescence increased from 0 to $4 \mathrm{~h}$ for all treatments, then reduced from hours 4 to 18 . PY signal in the 'midpiece' rose from 1 to $2 \mathrm{~h}$ of incubation, followed by a more robust increase in 'tail' and 'midpiece+tail' signal at 2 and $4 \mathrm{~h}$ (Fig. 2). At $4 \mathrm{~h}$ incubation, there was also a modest but nonsignificant increase in 'head' staining. The most common pattern in the sperm was the tail/midpiece+tail. At $2 \mathrm{~h}$, mCCM, mNCSU, and FERT-incubated sperm displayed significantly higher proportions of sperm with tail/ midpiece+tail PY signal compared with mSOF. This was reflective of the overall low proportions of PY signal in sperm incubated in mSOF. For example, at $2 \mathrm{~h}$ incubation, between 34 and $40 \%$ of sperm in mNCSU-23, mCCM, and FERT displayed PY signal, whereas only $20 \%$ and $6 \%$ of TRIS and mSOF, respectively, incubated sperm displayed any PY immunofluorescence.

As mNCSU-incubated sperm consistently displayed high motility, acrosome integrity, and robust PY signaling in extended culture, we selected it for additional comparison of less-stimulating or 'non-capacitating' (NC) conditions. A subset of samples (Supplementary Table 2, $n=2-5$ animals/treatment) were incubated under NC-mNCSU-23 compared with normal, capacitating mNCSU-23. Motility of sperm in NC-mNCSU-23 was significantly reduced compared with mNCSU-23 incubated sperm at hours 1, 2 , and 4 of incubation $(P<0.05$, Fig. 3A). Progesterone supplementation had no influence on percent motility for either media. Sperm in both media displayed a similar loss in acrosome integrity over $18 \mathrm{~h}$ culture $(P<0.05)$, with no 


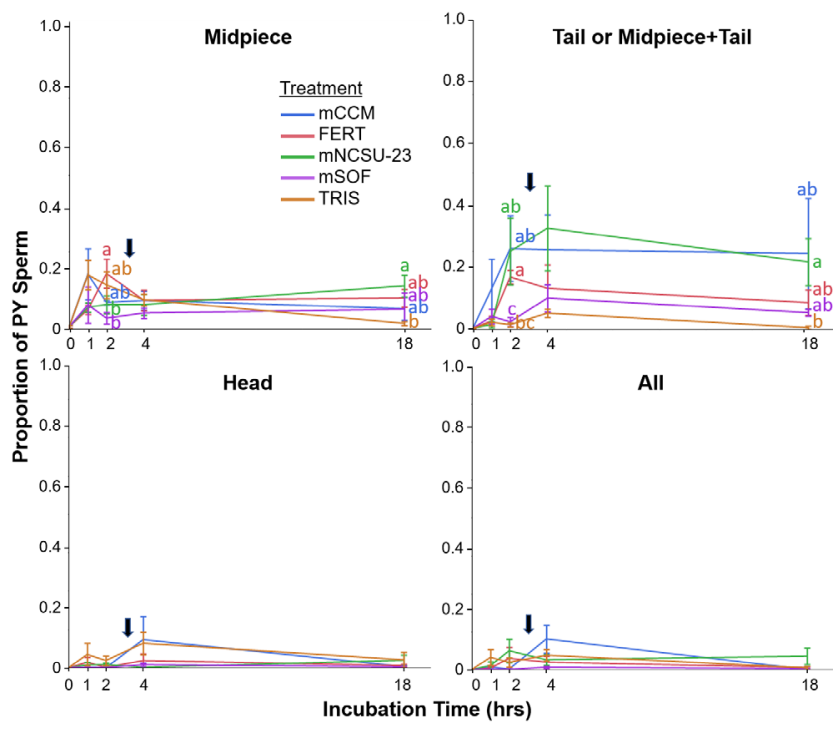

Figure 2 Patterns of tyrosine phosphorylation immunofluorescence (mean \pm S.E.M.) in red wolf sperm after $0,1,2,4$, and $18 \mathrm{~h}$ incubation in mCCM, FERT, mNCSU-23, mSOF, and TRIS medium. Letters indicate significant difference in pattern between treatment groups for each time point $(P<0.05)$ and black arrows denote time of progesterone treatment.

significant differences between progesterone treatment conditions (Fig. 3B). Incubation time and treatment group were significant in our model for PY patterns $(P<0.05)$, but not progesterone or its interaction with treatment. For example, for midpiece tyrosine phosphorylation, the signal rose in sperm exposed to both treatment conditions at $1-2 \mathrm{~h}$ of incubation but was significantly higher in NC-mNCSU-23 incubated sperm compared with mNCSU-23 ( $P<0.05$, Fig. 3C). A similar trend persisted in the other PY patterns (Supplementary Fig. 2), with the overall timeline of signal appearance during incubation similar for NC-mNCSU-23 as the earlier evaluated base medium (Fig. 2).

\section{Discussion}

The ability to maintain red wolf sperm in vitro and develop an understanding of capacitation is key to the application of various ART for species conservation. In this study, we sought to (1) identify a base medium supportive of red wolf sperm incubation, and (2) develop a capacitation protocol for red wolf sperm. We found that red wolf sperm motility and acrosome integrity is better maintained in mCCM, mNCSU-23, and mSOF in vitro compared with FERT and TRIS solutions. Induction of capacitation, based on patterns of tyrosine phosphorylation signal, occurred between 2 and $4 \mathrm{~h}$ in vitro. Results indicate that both mNCSU-23 and mCCM are promising sperm
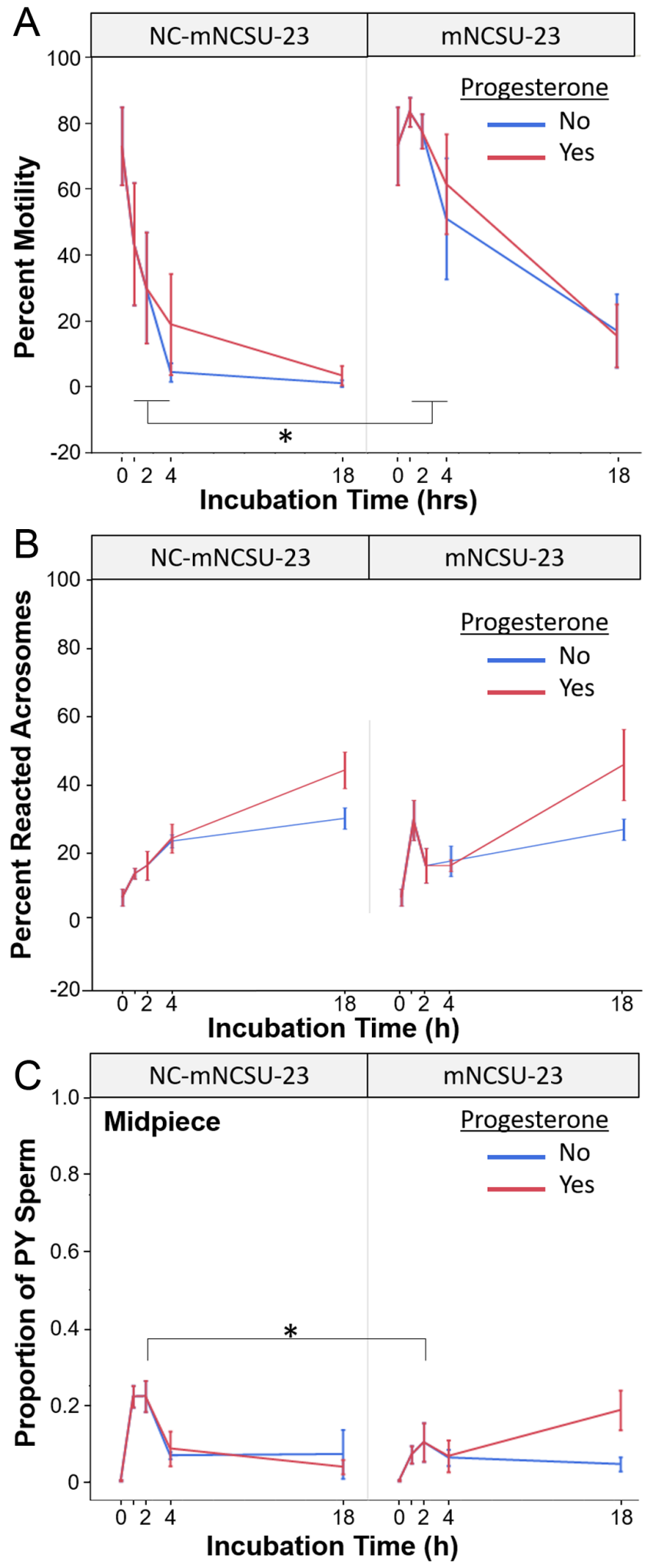

Figure 3 Percent motility (A), acrosome status (B), and proportion of midpiece tyrosine phosphorylation pattern $(C)$ of red wolf sperm incubated under non-capacitating mNCSU-23 (NC-mNCSU-23) or capacitating mNCSU-23 in the absence/presence of progesterone after $3 \mathrm{~h}$ in vitro. 
handling media, based on their ability to maintain sperm motility and acrosome integrity, and stimulate tyrosine phosphorylation. Our subsequent evaluation of mNCSU23 indicates additional optimizations are necessary to improve capacitated red wolf sperm's ability to undergo acrosome exocytosis. Nevertheless, these results represent a key first step toward the recapitulation of capacitation in vitro, necessary to produce fertilization-competent sperm for IVF work and to evaluate the quality of post-thaw semen in the future.

In the current study, red wolf sperm characteristics were consistent with what has previously been reported for the population from 1990 to 2004 (Lockyear et al. 2016), with motility following electroejaculation $~ 70 \%$ and concentration around 70 million sperm $/ \mathrm{mL}$. In much of the previous red wolf semen incubation research thus far, the focus has been on post-thaw motility (Lockyear et al. 2009, Franklin et al. 2018). Only one study to date has incubated freshly collected red wolf semen, detailing a decrease in motility from $~ 70$ to $55 \%$ after 4 $\mathrm{h}$ in vitro in Tyrode's albumin lactate pyruvate at room temperature or $0^{\circ} \mathrm{C}$ (Goodrowe et al. 2001). Interestingly, motility of sperm held at $37^{\circ} \mathrm{C}$ in this study fell to $\sim 40 \%$ in the same time frame, and dropped to zero by $10 \mathrm{~h}$ in vitro incubation, whereas those stored at the lower, nonphysiological temperatures maintained $\sim 30 \%$ motility for $10 \mathrm{~h}$. As such, we were interested in evaluating different base medium for the maintenance of fresh red wolf sperm function at physiological temperatures. While we still observed a reduction from $74 \%$ total motile sperm to $55 \%$ after $4 \mathrm{~h}$ in vitro in $\mathrm{mCCM}$ and mNCSU-23 incubated medium at $38^{\circ} \mathrm{C}$ at $5 \% \mathrm{CO}_{2}$ in the current study, this is in-line with the better-sustained motility levels observed in ambient or chilled samples previously (Goodrowe et al. 2001, Franklin et al. 2018). Our results indicate, therefore, that these culture media and/or conditions support the maintenance of red wolf sperm under physiological temperatures in vitro.

The appearance of hyperactive motility at $4 \mathrm{~h}$ incubation, in combination with elevated midpiece and tail tyrosine phosphorylation patterns, indicates that red wolf sperm capacitation is initiated between 2 and $4 \mathrm{~h}$ in vitro. This is similar to the finding in domestic dog sperm incubated in $\mathrm{mCCM}$, with the proportion of sperm with hyperactive motility steadily increasing during 1-7 h of incubation (from $20 \%$ at $1 \mathrm{~h}$ to $80 \%$ at $7 \mathrm{~h}$ ) (Kawakami et al. 1998, Kawakami et al. 2000). Patterns of tyrosine phosphorylation in the red wolf were also consistent with a previous study in the domestic dog (Petrunkina et al. 2003). In the dog, initial PY signal is observed in the midpiece within the first hour of incubation, followed by the signal in the tail between hours 1 and 3 , and strong signal in the head region primarily after $3 \mathrm{~h}$ incubation and a subsequent significant decrease in tail phosphorylation after $6 \mathrm{~h}$ (Petrunkina et al. 2003). While the same overall pattern was observed in the present study, sperm incubated in $\mathrm{mSOF}$ and TRIS displayed less robust tail and midpiece+tail signals. As the TRIS medium was composed only of the base buffer (Franklin et al. 2018), with no components added to stimulate capacitation (i.e. BSA or bicarbonate), the lack of signal was not unexpected.

While progesterone itself has been indicated as stimulating capacitation in some species, including the human (de Lamirande et al. 1998) and pig (Barboni et al. 1995), it did not appear to have an impact on capacitation in the red wolf in our study. Notably, there were no statistically significant differences in motility or tyrosine phosphorylation patterns between $\mathrm{P} 4$ and no-P4 treated mNCSU-23 incubated sperm at 4 or $18 \mathrm{~h}$ of incubation (Fig. 3 and Supplementary Fig. 2). In non-capacitating mNCSU, the decline in sperm motility/viability in both $\mathrm{P} 4$ and no-P4 groups may have been due to poor $\mathrm{pH}$ buffering in the absence of bicarbonate, or a need for higher levels of BSA to support red wolf sperm survival in vitro. It is possible that, in mNCSU-23 or a more balanced NC-mNCSU-23, the inclusion of additional time-points (i.e. 6 or 8 h) might reveal a delayed effect of progesterone, which should be evaluated in future studies. Alternatively, the red wolf may be similar to the bovine, wherein P4 has been shown to stimulate acrosome reaction but not capacitation in fresh epididymal sperm (Thérien \& Manjunath 2003). While progesterone receptors have not been localized on red wolf sperm, work in the domestic dog demonstrated no signal using antibodies against progesterone receptor in un-capacitated sperm, but strong signal over the acrosomal region in capacitated samples (Wu et al. 2005). Therefore, we postulate that progesterone is not a key stimulant of sperm capacitation in the red wolf.

Surprisingly, we did not observe an appreciable increase in acrosome-reacted sperm following stimulation with $6 \mu \mathrm{g} / \mathrm{mL}(19 \mu \mathrm{M})$ progesterone after $3 \mathrm{~h}$ incubation (Fig. 1B), despite evidence of capacitation progression. While TRIS- incubated sperm did display an increase in reacted acrosomal membranes from 2 to $4 \mathrm{~h}$ incubation, this was mirrored by a significant decline in motile sperm (Fig. 1A) and thereby likely indicative of TRIS-incubated sperm becoming non-viable. Based on domestic dog work (Sirivaidyapong et al. 1999, Brewis et al. 2001), we anticipated significant induction of acrosomal exocytosis

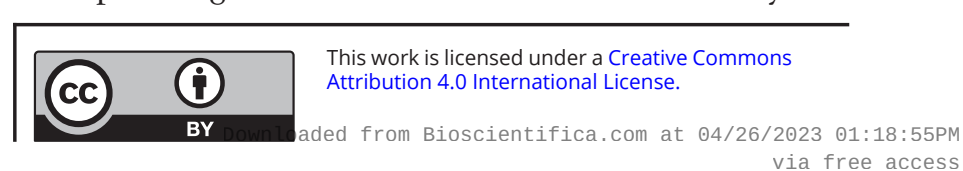


in progesterone-treated sperm within 20 min to $1 \mathrm{~h}$ of incubation, assuming they had achieved capacitation. Thus, in the subset of samples incubated under 'noncapacitating' mNCSU-23 and mNCSU-23, we expected a robust acrosome reaction to progesterone in the latter compared with the former medium at $4 \mathrm{~h}$ incubation. Instead, we observed similar acrosome patterns for both media with or without progesterone, suggesting that either red wolf sperm capacitation was not sufficiently achieved after $3 \mathrm{~h}$ incubation and/or the use of progesterone for stimulation of acrosomal exocytosis was not optimal for this species. The $\sim 19 \mu \mathrm{M}$ concentration of progesterone utilized here was slightly higher than what has previously been utilized for domestic dog sperm, ranging typically from $10 \mu \mathrm{M}$ (Sirivaidyapong et al. 1999, Deppe et al. 2010) to $13 \mu \mathrm{M}$ (Brewis et al. 2001); however, it is in range with dog follicular fluid, which is $\sim 2.6 \mu \mathrm{g} / \mathrm{mL}(\sim 8$ $\mu \mathrm{M})$ before the luteinizing hormone ( $\mathrm{LH})$ surge, rising to $\sim 11.7 \mu \mathrm{g} / \mathrm{mL}(\sim 37 \mu \mathrm{M})$ post-LH surge (Fahiminiya et al. 2010). As the progesterone concentration in the oviductal fluid around the time of fertilization in canid species is not yet known, it is possible that the concentrations applied here were not optimal for induction of acrosome exocytosis. Alternatively, a previous study in the silver fox (Vulpes vulpes) has reported that sperm require $5-8 \mathrm{~h}$ to capacitate and subsequently successfully penetrate fox ova (Feng et al. 1994). Moving forward, studies should determine if red wolf sperm require longer incubation for in vitro capacitation prior to stimulation to induce acrosome exocytosis, more frequent evaluation following stimulation to assess effects, or if alternative/additional stimulants (i.e. zona pellucida proteins (Brewis et al. 2001)) are more physiologically relevant than progesterone for induction of the acrosome reaction in this species.

Similarly, the investigation into the effects of specific medium components may be warranted to further optimize the capacitation of red wolf sperm. For example, both mNCSU and mCCM contained relatively high levels of glucose (5.55 and $2.78 \mathrm{mM}$, respectively), compared with $\mathrm{mSOF}$ and FERT $(1.50$ and $0.0 \mathrm{mM}$, respectively see Supplementary Table 1). Glucose supplementation has been demonstrated to extend the survivability of human sperm (Amaral et al. 2011) and induce hyperactivation-like changes in motility in domestic dog sperm (Rigau et al. 2001). We postulate that these higher glucose concentrations in mNCSU and mCCM may have had a similar supportive effect in the red wolf sperm. This effect was likely absent in TRIS-incubated sperm owing to the lack of other supportive components, such as BSA. Similarly, the absence of additional amino acids in mCCM compared with mNCSU did not appear to significantly influence any of the sperm metrics evaluated in this study. Though taurine and hypotaurine have been demonstrated to improve mammalian sperm motility in vitro (Meizel et al. 1980, Alvarez \& Storey 1983), the lack of significant differences between mCCM and mNCSUincubated sperm suggests these factors may not be as influential in the red wolf. Still, with the identification of a supportive base medium here, we can now adjust these specific components to better understand the mechanisms and improve red wolf sperm capacitation.

To date, few studies have specifically assessed sperm capacitation in non-domestic canid species. The paucity of work in this field is due in part to the difficulty in obtaining fresh semen samples to study, particularly in endangered canid species. Nevertheless, with 11 of the 36 extant species of canid listed as either endangered or near threatened (IUCN 2020), the development of genome preservation and assisted reproductive technologies for canids is becoming increasingly important to species survival (Luvoni et al. 2006). In this study, we have identified two base media (mCCM and mNCSU-23) capable of supporting in vitro incubation of red wolf sperm at physiological temperatures. Further, we have described patterns of tyrosine phosphorylation during the early stages of capacitation in the red wolf. While further studies are necessary to develop in vitro capacitation for application to IVF or for the evaluation of sperm fertilizing capacity post-thaw, our findings represent an important step in the development of assisted reproductive technologies in the critically endangered red wolf.

\section{Supplementary materials}

This is linked to the online version of the paper at https://doi.org/10.1530/ RAF-20-0042.

\section{Declaration of interest}

The authors declare that there is no conflict of interest that could be perceived as prejudicing the impartiality of the research reported.

\section{Funding}

This work was supported by the Volgenau Foundation, Brandt Foundation, Point Defiance Zoo and Aquarium's Dr Holly Reed Conservation Fund, the Friends of the National Zoo (FONZ), and the Smithsonian Institution.

\section{Author contribution statement}

J B N, M d A M M F and N S conceived the study. J B N, M d A M M F, and S $\mathrm{H}$ K performed the experiments. J $\mathrm{N}$ analyzed the data. J B N, M d A M M F, S H K, and N S wrote the paper. 


\section{Acknowledgements}

The authors would like to thank the following institutions and their staff: Chattanooga Nature Riding and Reflection Center (Chattanooga, TN), North Carolina Zoo (Asheboro, NC), Wolf Conservation Center (South Salem, NY), Salisbury Zoo (Salisbury, MD), and the Mill Mountain Zoo (Roanoke, VA). The authors would also like to thank the AZA red wolf Species Survival Plan coordinators, Chris Lasher and Will Waddell.

\section{References}

Alvarez JG \& Storey BT 1983 Taurine, hypotaurine, epinephrine and albumin inhibit lipid peroxidation in rabbit spermatozoa and protect against loss of motility. Biology of Reproduction 29 548-555. (https:// doi.org/10.1095/biolreprod29.3.548)

Amaral A, Paiva C, Baptista M, Sousa AP \& Ramalho-Santos J 2011 Exogenous glucose improves long-standing human sperm motility, viability, and mitochondrial function. Fertility and Sterility 96 848-850. (https://doi.org/10.1016/j.fertnstert.2011.07.1091)

Amstislavsky S, Lindeberg H \& Luvoni G 2012 Reproductive technologies relevant to the genome resource bank in Carnivora. Reproduction in Domestic Animals 47 164-175. (https://doi. org/10.1111/j.1439-0531.2011.01886.x)

Asa CS, Bauman K, Callahan P, Bauman J, Volkmann DH \& Jöchle W 2006 GnRH-agonist induction of fertile estrus with either natural nating or artificial insemination, followed by birth of pups in gray wolves (Canis lupus). Theriogenology 66 1778-1782. (https://doi. org/10.1016/j.theriogenology.2006.01.035)

Austin CR 1952 The 'capacitation' of the mammalian sperm. Nature 170 326. (https://doi.org/10.1038/170326a0)

Barboni B, Mattioli M \& Seren E 1995 Influence of progesterone on boar sperm capacitation. Journal of Endocrinology 144 13-18. (https:// doi.org/10.1677/joe.0.1440013)

Bates D, Mächler M, Bolker B \& Walker S 2014 Fitting linear mixed-effects models using lme4. arXiv Preprint ArXiv:1406.5823.

Blanco JM, Wildt DE, Höfle U, Voelker W \& Donoghue AM 2009 Implementing artificial insemination as an effective tool for ex situ conservation of endangered avian species. Theriogenology $\mathbf{7 1}$ 200-213. (https://doi.org/10.1016/j.theriogenology.2008.09.019)

Brewis IA, Morton IE, Moore HDM \& England GCW 2001 Solubilized zona pellucida proteins and progesterone induce calcium influx and the acrosome reaction in capacitated dog spermatozoa. Molecular Reproduction and Development 60 491-497. (https://doi. org/10.1002/mrd.1114)

Buffone MG, Wertheimer EV, Visconti PE \& Krapf D 2014 Central role of soluble adenylyl cyclase and cAMP in sperm physiology. Biochimica et Biophysica Acta 1842 2610-2620. (https://doi. org/10.1016/j.bbadis.2014.07.013)

Chang MC 1951 Fertilizing capacity of spermatozoa deposited into the fallopian tubes. Nature 168 697-698. (https://doi. org/10.1038/168697b0)

Comizzoli P, Crosier AE, Songsasen N, Gunther MS, Howard JG \& Wildt DE 2009 Advances in reproductive science for wild carnivore conservation. Reproduction in Domestic Animals $\mathbf{4 4}$ (Supplement 2) 47-52. (https://doi.org/10.1111/j.14390531.2009.01373.x)

Cross NL 1998 Role of cholesterol in sperm capacitation. Biology of Reproduction 59 7-11. (https://doi.org/10.1095/biolreprod59.1.7)

de Lamirande E, Harakat A \& Gagnon C 1998 Human sperm capacitation induced by biological fluids and progesterone, but not by NADH or NADPH, is associated with the production of superoxide anion. Journal of Andrology 19 215-225. (https://doi. org/10.1002/j.1939-4640.1998.tb01991.x)
Demarco IA, Espinosa F, Edwards J, Sosnik J, De La VegaBeltran JL, Hockensmith JW, Kopf GS, Darszon A \& Visconti PE 2003 Involvement of a Na+/HCO-3 cotransporter in mouse sperm capacitation. Journal of Biological Chemistry 278 7001-7009. (https://doi.org/10.1074/jbc.M206284200)

Deppe M, Risopatrón J \& Sánchez R 2010 Trypsin and chymotrypsin are involved in the progesterone-induced acrosome reaction in canine spermatozoa. Reproduction in Domestic Animals 45 453-457. (https://doi.org/10.1111/j.1439-0531.2008.01225.x)

Fahiminiya S, Reynaud K, Labas V, Batard S, ChastantMaillard S \& Gérard N 2010 Steroid hormones content and proteomic analysis of canine follicular fluid during the preovulatory period. Reproductive Biology and Endocrinology 8 132. (https://doi. org/10.1186/1477-7827-8-132)

Farstad W, Fougner JA \& Torres CG 1992 The optimum time for single artificial insemination of blue fox vixens (Alopex lagopus) with frozen-thawed semen from silver foxes (Vulpes vulpes). Theriogenology 38 853-865. (https://doi.org/10.1016/0093$691 x(92) 90161-j)$

Faust L, Harrison R, Waddell W, Long S \& Traylor-Holzer K 2016 Red wolf (Canis rufus) population viability analysis- Report to US Fish and Wildlife Service, Lincoln Park Zoo, Chicago, IL, USA.

Feng HL, Liu JM, Wen XH, Wang J, Sun QY \& Qin PC 1994 Follicular oocyte maturation and sperm penetration in vitro in the silver fox (Vulpes vulpes). Animal Reproduction Science 36 163-170. (https://doi.org/10.1016/0378-4320(94)90063-9)

Ferraz MAMM, Rho HS, Hemerich D, Henning HHW, van Tol HTA, Hölker M, Besenfelder U, Mokry M, Vos PLAM, Stout TAE et al. 2018 An oviduct-on-a-chip provides an enhanced in vitro environment for zygote genome reprogramming. Nature Communications 9 4934. (https://doi.org/10.1038/s41467-018-07119-8)

Ferraz MDAMM, Nagashima JB, Noonan MJ, Crosier AE \& Songsasen N 2020 Oviductal extracellular vesicles improve post-thaw sperm function in red wolves and cheetahs de Almeida. International Journal of Molecular Sciences 21 3733. (https://doi. org/10.3390/ijms21103733)

Franklin AD, Waddell WT \& Goodrowe KL 2018 Red wolf (Canis rufus) sperm quality and quantity is affected by semen collection method, extender components, and post-thaw holding temperature. Theriogenology 116 41-48. (https://doi.org/10.1016/j theriogenology.2018.05.007)

Goodrowe KL, Hay MA, Platz CC, Behrns SK, Jones MH \& Waddell WT 1998 Characteristics of fresh and frozen-thawed red wolf (Canis rufus) spermatozoa. Animal Reproduction Science 53 299-308. (https://doi.org/10.1016/s0378-4320(98)00119-5)

Goodrowe KL, Mastromonaco GF, Walker SL, Bateman HL, Ryckman DP, Platz CC \& Waddell WT 2001 In vitro maintenance, cooling and cryopreservation of red wolf (Canis rufus) spermatozoa. Journal of Reproduction and Fertility: Supplement 57 387-392.

Hewitt DA \& England GCW 1999 Synthetic oviductal fluid and oviductal cell coculture for canine oocyte maturation in vitro. Animal Reproduction Science 55 63-75. (https://doi.org/10.1016/s03784320(98)00162-6)

Howard JG, Lynch C, Santymire RM, Marinari PE \& Wildt DE 2016 Recovery of gene diversity using long-term cryopreserved spermatozoa and artificial insemination in the endangered black-footed ferret. Animal Conservation 19 102-111. (https://doi. org/10.1111/acv.12229)

Huang Y, Li D, Zhou Y, Zhou Q, Li R, Wang C, Huang Z, Hull v \& Zhang $\mathbf{H} 2012$ Factors affecting the outcome of artificial insemination using cryopreserved spermatozoa in the giant panda (Ailuropoda melanoleuca). Zoo Biology 31 561-573. (https://doi. org/10.1002/zoo.20421)

IUCN 2020 The IUCN Red list of threatened species. (available at: www. iucnredlist.org). Downloaded 12 July 2020.

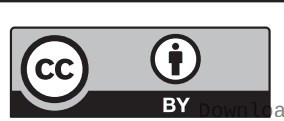


Kawakami E, Hori T \& Tsutsui T 1998 Induction of dog sperm capacitation by oviductal fluid. Journal of Veterinary Medical Science $\mathbf{6 0}$ 197-202. (https://doi.org/10.1292/jvms.60.197)

Kawakami E, Arai T, Oishi I, Hori T \& Tsutsui T 2000 Induction of dog sperm capacitation by glycosaminoglycans and glycosaminoglycan amounts of oviductal and uterine fluids in bitches. Journal of Veterinary Medical Science 62 65-68. (https://doi. org/10.1292/jvms.62.65)

Koehler JK, Platz CC, Waddell W, Jones MH \& Behrns S 1998 Semen parameters and electron microscope observations of spermatozoa of the red wolf, Canis rufus. Journal of Reproduction and Fertility 114 95-101. (https://doi.org/10.1530/jrf.0.1140095)

Lasher C 2020 Status of the SSP. In American Red Wolf SSP Master Planning Meeting.

Lockyear KM, Goodrowe KL, Waddell WT \& MacDonald SE 2009 Comparison of different osmolalities and egg-yolk composition in processing media for the cryopreservation of red wolf (Canis rufus) sperm. Theriogenology 71 469-479. (https://doi.org/10.1016/j. theriogenology.2008.07.030)

Lockyear KM, MacDonald SE, Waddell WT \& Goodrowe KL 2016 Investigation of captive red wolf ejaculate characteristics in relation to age and inbreeding. Theriogenology 86 1369-1375. (https://doi. org/10.1016/j.theriogenology.2016.04.080)

Luvoni GC, Chigioni S \& Beccaglia M 2006 Embryo production in dogs: from in vitro fertilization to cloning. Reproduction in Domestic Animals $\mathbf{4 1}$ 286-290. (https://doi.org/10.1111/j.1439-0531.2006.00704.x)

Mahi CA \& Yanagimachi R 1978 Capacitation, acrosome reaction, and egg penetration by canine spermatozoa in a simple defined medium. Gamete Research 1 101-109. (https://doi.org/10.1002/ mrd.1120010203)

Meizel S, Lui CW, Working PK \& Mrsny RJ 1980 Taurine and hypotaurine: their effects on motility, capacitation and the acrosome reaction of hamster sperm in vitro and their presence in sperm and reproductive tract fluids of several mammals. Development, Growth and Differentiation 22 483-494. (https://doi.org/10.1111/j.1440169X.1980.00483.x)

Mossotti R 2017 World's first Mexican wolf pup born from artificially inseminated frozen/thawed semen. In Partnership of Government, Institutions Achieved Reproductive Breakthrough for Critically Endangered Species 3. Eureka, MO: Endangered Wolf Center.

Nagashima JB, Sylvester SR, Nelson JL, Cheong SH, Mukai C, Lambo C, Flanders JA, Meyers-Wallen VN, Songsasen N \& Travis AJ 2015 Live births from domestic dog (Canis familiaris) embryos produced by in vitro fertilization. PLOS ONE 10 e0143930. (https://doi.org/10.1371/journal.pone.0143930)

Nyberg K 1980 Crossing blue fox (Alopex lagopus) to red fox (Vulpes vulpes) using artificial insemination. Nordisk Veterinaermedicin 32 132-138.
Parrish JJ 2014 Bovine in vitro fertilization: in vitro oocyte maturation and sperm capacitation with heparin. Theriogenology 81 67-73. (https://doi.org/10.1016/j.theriogenology.2013.08.005)

Petrunkina AM, Simon K, Gunzel-Apel AR \& Topfer-Petersen E 2003 Specific order in the appearance of protein tyrosine phosphorylation patterns is functionally coordinated with dog sperm hyperactivation and capacitation. Journal of Andrology 24 423-437. (https://doi.org/10.1002/j.1939-4640.2003.tb02691.x)

Petters RM 1992 In vitro culture of early stage embryos from livestock. Tissue Culture Research Communications 11 305-313. (https://doi org/10.11418/jtca1981.11.3_305)

Petters RM \& Wells KD 1993 Culture of pig embryos. Journal of Reproduction and Fertility: Supplement 48 61-73.

Pinheiro J, Bates D, DebRoy S, Sarkar D \& Team RC 2012 nlme: linear and nonlinear mixed effects models. R package version 3 .

Rigau T, Farré M, Ballester J, Mogas T, Pena A \& RodriguezGil JE 2001 Effects of glucose and fructose on motility patterns of dog spermatozoa from fresh ejaculates. Theriogenology 56 801-815. (https://doi.org/10.1016/s0093-691x(01)00609-4)

Roldan ER, Murase T \& Shi QX 1994 Exocytosis in spermatozoa in response to progesterone and zona pellucida. Science 266 1578-1581. (https://doi.org/10.1126/science.7985030)

Sirivaidyapong S, Bevers MM \& Colenbrander B 1999 Acrosome reaction in dog sperm is induced by a membrane-localized progesterone receptor. Journal of Andrology 20 537-544. (https://doi. org/10.1002/j.1939-4640.1999.tb02553.x)

Thérien I \& Manjunath P 2003 Effect of progesterone on bovine sperm capacitation and acrosome reaction. Biology of Reproduction 69 1408-1415. (https://doi.org/10.1095/biolreprod.103.017855)

Visconti PE, Bailey JL, Moore GD, Pan D, Olds-Clarke P \& Kopf GS 1995 Capacitation of mouse spermatozoa. I. Correlation between the capacitation state and protein tyrosine phosphorylation. Development 121 1129-1137.

Visconti PE, Stewart-Savage J, Blasco A, Battaglia L, Miranda $P$, Kopf GS \& Tezón JG 1999 Roles of bicarbonate, cAMP, and protein tyrosine phosphorylation on capacitation and the spontaneous acrosome reaction of hamster sperm. Biology of Reproduction $\mathbf{6 1}$ 76-84. (https://doi.org/10.1095/biolreprod61.1.76)

Wassarman PM 1999 Mammalian fertilization: molecular aspects of gamete adhesion, exocytosis, and fusion. Cell 96 175-183. (https:// doi.org/10.1016/s0092-8674(00)80558-9)

Wu JT, Tsai PS, Lee SL \& Cheng FP 2005 Characterisation of the progesterone receptor on canine spermatozoa. Reproduction, Fertility, and Development 17 733-741. (https://doi.org/10.1071/rd05074)

Yanagimachi R 1970 The movement of golden hamster spermatozoa before and after capacitation. Journal of Reproduction and Fertility 23 193-196. (https://doi.org/10.1530/jrf.0.0230193)

Received in final form 5 November 2020

Accepted 20 November 2020

Accepted Manuscript published online 20 November 2020 https://raf.bioscientifica.com

https://doi.org/10.1530/RAF-20-0042 (c) 2020 The authors Published by Bioscientifica Ltd

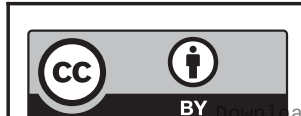

This work is licensed under a Creative Commons Attribution 4.0 International License. 